\title{
Preparation of Transmission Electron Microscope Specimens from Ultra-Fine Fibers by a FIB Technique
}

Jian Li, V. Y. Gertsman, and J. Lo, Materials Technology Laboratory, CANMET, 568 Booth St. Ottawa, Ontario, Canada, K1A 0G1

Focused ion beam (FIB) microscopes have become a powerful tool in transmission electron microscope (TEM) specimen preparation [1,2]. These techniques have already been well established. In the recent years, a more advanced "lift-out" technique has proved to be a very powerful one in the cases where mechanical pre-preparations are difficult or impossible [1]. The advantage of the lift-out technique is that TEM specimens can be made directly from the bulk specimen inside a FIB microscope. However, a major disadvantage is that once the TEM sample is made, it cannot be re-thinned if needed. The "lift-out" technique has been further improved by an "H-bar lift-out" technique [3]. Instead of lifting out an electron transparent specimen, a much thicker specimen (typically 3-4 $\mu \mathrm{m}$ in thickness) is lifted out from the bulk. This specimen containing the feature of interest is then mounted onto a large carrier using a micromanipulator and then thinned in a FIB microscope.

In some cases, currently available FIB-TEM sample preparation techniques become inadequate. In our current study, we need to examine a cross-section of a nickel coated carbon fiber with a diameter of $8 \mu \mathrm{m}$ in TEM. Neither the conventional nor "H-bar lift-out" techniques are capable of producing a TEM specimen of a specific fiber with the whole cross-section electron transparent.

Consequently, we have modified the lift-out technique to allow the preparation of an $8 \mu \mathrm{m}$ diameter electron-transparent fiber specimen. A $3 \mathrm{~mm}$ copper aperture disk with $\sim 800 \mu \mathrm{m}$ inner diameter is cut to about its $2 / 3$ of its original size using a razor blade. A small amount of epoxy is applied onto the inner edge of the copper ring which is used as the carrier for the fiber specimen. The nickel coated carbon fibers of $8 \mu \mathrm{m}$ in diameter are cut to about $4 \mathrm{~mm}$ in length. A micromanipulator is used to mount the desired fiber onto the edge of the copper grid as shown in Figures 1 and 2. The specimen is then loaded into a Micrion 2500 FIB system for ion beam cutting and thinning. Figure 3 shows the complete fiber cross-section prior to final FIB thinning. The relatively thick FIB deposited tungsten layer on top of the fiber protects the upper edge from $\mathrm{Ga}$ ion beam erosion during FIB thinning and also serves as an additional support and conductive path for the thin specimen. Figure 4 shows a TEM image of the finished cross-section of the entire fiber.

The advantages of this technique are: 1). The whole cross-section of the fiber can be made electron transparent for TEM observation if required. 2). Very quick and straightforward to prepare. 3) Compared to the conventional lift-out technique, this technique reduces the risk of potential mechanical damage by the micromanipulator when the specimen is thin and fragile.

\section{Acknowledgments}

The authors would like to thank Dr. L. Weaver and Mr. M. W. Phaneuf of FIBICS Incorporated for useful discussions. Technical advices from Dr. Quan Yang of INCO Technical Services Ltd. and Dr. Sylvie Dionne of CANMET-MTL are greatly appreciated. 
References

[1] L.A. Giannuzzi and F.A. Stevie, Micron, 30, (issue 3) (1999) p.197.

[2] J.M. Cairney and P.R. Munroe, Materials Characterization, 46, (2001) p.297.

[3] R.J. Patterson et al., Microscopy and Microanalysis (2002) p.566.

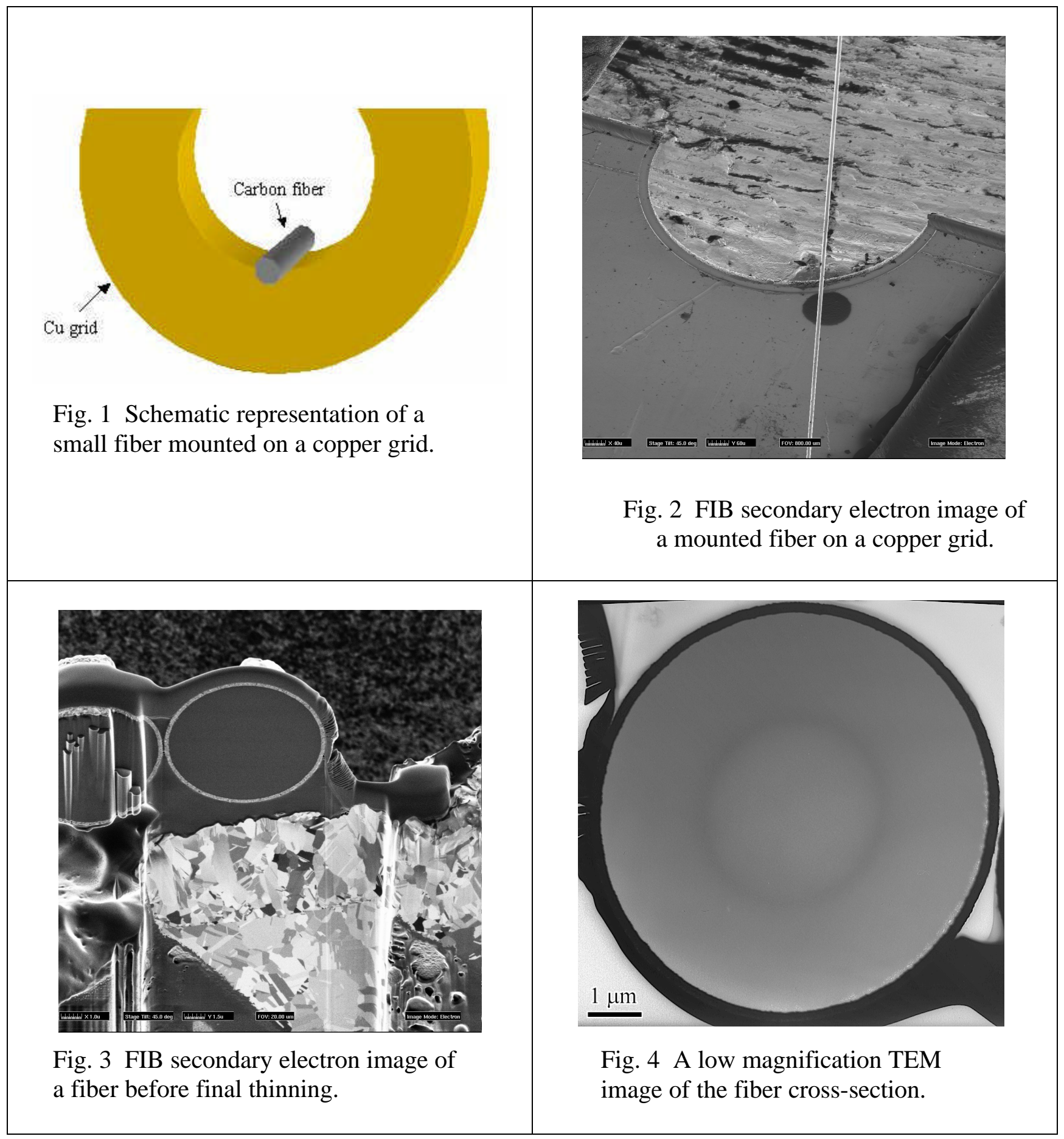

